\title{
Konsuminteresse wecken
}

In vielen Handels- und Dienstleistungsbranchen lässt sich eine steigende Konsumzurückhaltung erkennen. Ein Grund für die sich ausbreitende Shopping-Lethargie liegt im hohen Konsumniveau. „Konsumsattheit“ kennen wir alle: Unser Kleiderschrank ist brechend voll, wir besitzen mehr Fernsehgeräte im eigenen Haushalt als Zuschauer und fürchten die Gefahr der Produktveralterung aufgrund immer kürzerer Produktlebenszyklen. Aber auch das Überangebot an Produktalternativen und Vertriebskanälen, die Austauschbarkeit im Angebot sowie eine nicht mehr nachvollziehbare „Aktionitis“ mit immer höheren Preisnachlässen überfordern uns. Wer hat immer Zeit und geschweige denn Lust, sich im Labyrinth eines überbordernden Angebotsdschungels zurechtzufinden? Zudem gewinnt das Sparverhalten privater Haushalte in Zeiten anhaltender Wirtschafts- und Schuldenkrisen an Relevanz. Smart-Shopping kommt immer stärker in Mode. Die daraus entstehenden Gefahren sind weitreichend: Umsatzrückgang, Kosteneinsparungen bis hin zu Mitarbeiterabbau und teilweise gar die Zahlungsunfähigkeit zählen dazu.

Für Unternehmen ergibt sich daraus die Aufgabe, gekonnt auf die zunehmende Konsumverdrossenheit zu reagieren. Neben der permanenten Rationalisierung und Produktivitätssteigerung steht das Marketing vor der großen Herausforderung, Kundeninteresse überzeugender und nachhaltiger zu wecken. Altbekannte, aber auch neue Marketingansätze stehen dafür zur Verfügung. Ein bisher bewährter Ansatz verliert jedoch an Wirkungskraft: Mit noch höheren Rabatten lässt sich zwar kurzfristig der Umsatz ankurbeln, aber langfristig stellen sich nach den Erfahrungen vieler Unternehmen Umsatzeinbußen aufgrund tieferer Durchschnittsumsätze ein. Um das Kundeninteresse nachhaltig zu wecken, braucht es mehr als wohlklingende Marketingkampagnen, die häufig mehr versprachen, als sie halten konnten. Dafür ist ein hohes $\mathrm{Ma} ß$ an Innovation und Kreativität erforderlich.

Die Beiträge dieser Ausgabe beleuchten aus unterschiedlichen Blickwinkeln die anspruchsvolle Aufgabe, das Interesse der Konsumenten in Zeiten einer stagnierenden Wirtschaft zu wecken. Um ein grundlegendes Verständnis für sparende Konsumenten zu schaffen, werden Auslöser der Konsumzurückhaltung und die Auswirkungen auf das Einkaufsverhalten näher untersucht. Zwei Beiträge zeigen auf,

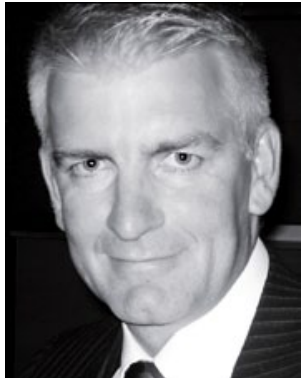

Prof. Dr. Thomas Rudolph

ist Direktor des Forschungszentrums für

Handelsmanagement (Institute of Retail

Management IRM-HSG) an der

Universität St. Gallen (HSG).

E-Mail: thomas.rudolph@unisg.ch

wie der Umsatz mit sparenden Konsumenten in Spanien und der Schweiz angekurbelt werden kann. Aber auch neue Serviceleistungen im Internet, der gekonnte Einsatz von Duft in der Markenführung sowie der Aufbau von Erlebniswelten durch Sponsoring vermögen Kundeninteresse wecken. Dabei muss das Marketing neue Wege gehen. Eine Erfolgsgeschichte mit konkreten Hinweisen für das Wecken von nachhaltigem Kundeninteresse zeigt das Interview mit Götz Werner, dem Gründer von dm Drogeriemarkt, in dieser Ausgabe der Marketing Review St. Gallen auf. Während die bisher genannten Beiträge den Business-to-Consumer-Markt fokussieren, beleuchtet ein weiterer Artikel dieser Ausgabe die Herausforderungen an den Schnittstellen zwischen Marketing und Verkauf im Business-to-Business-Bereich.

Wir hoffen, Ihr Interesse an diesem spannenden Thema geweckt zu haben und wünschen eine inspirierende Lektüre.

Ihr

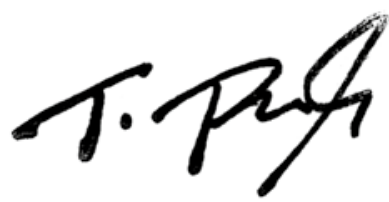

Thomas Rudolph 